UCRL-ID-131921

\title{
Elastic Properties of Large Tow 2-D Braided Composites by Numerical and Analytical Methods ${ }^{1}$
}

Thao D. Nguyen

Edward Zywicz

September 1, 1998

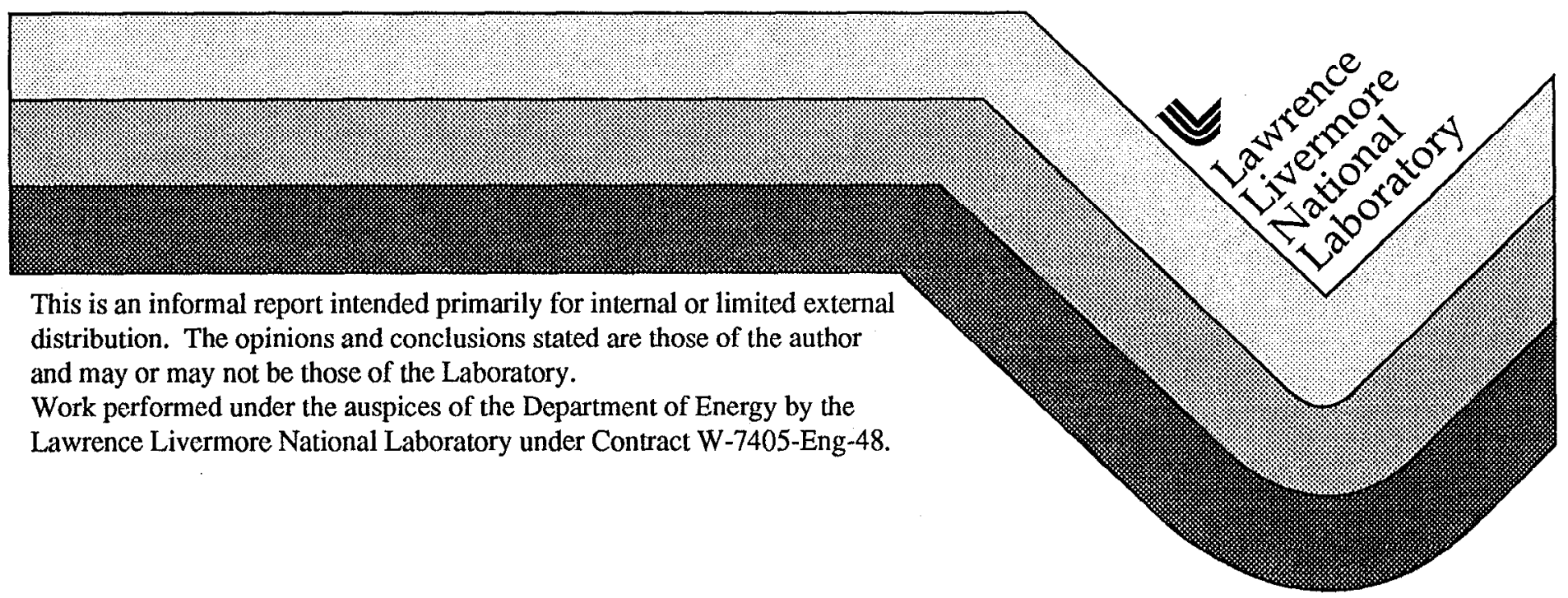




\section{DISCLAIMER}

This document was prepared as an account of work sponsored by an agency of the United States Government. Neither the United States Government nor the University of California nor any of their employees, makes any warranty, express or implied, or assumes any legal liability or responsibility for the accuracy, completeness, or usefulness of any information, apparatus, product, or process disclosed, or represents that its use would not infringe privately owned rights. Reference herein to any specific commercial product, process, or service by trade name, trademark, manufacturer, or otherwise, does not necessarily constitute or imply its endorsement, recommendation, or favoring by the United States Govemment or the University of Califomia. The views and opinions of authors expressed herein do not necessarily state or reflect those of the United States Government or the University of Califomia, and shall not be used for advertising or product endorsement purposes.

This report has been reproduced directly from the best available copy.

Available to DOE and DOE contractors from the Office of Scientific and 'lechnical Information P.O. Box 62, Oak Ridge, TN 37831

Prices available from (615) 576-8401, FTS 626-8401

Available to the public from the

National Technical Information Service

U.S. Department of Commerce 5285 Port Royal Rd., Springfield, VA 22161 


\title{
Elastic Properties of Large Tow 2-D Braided Composites by Numerical and Analytical Methods ${ }^{1}$
}

\author{
Thao D. Nguyen \\ Edward Zywicz \\ University of California \\ Lawrence Livermore National Laboratory \\ Livermore, CA 94556
}

September 9, 1998

\begin{abstract}
The homogenized extensional and flexural properties of a large tow, twodimensional, braided carbon-fiber composite lamina were evaluated using analytical and numerical methods. The plane-stress composite lamina was assumed to be periodic in its plane and was modeled with a single representative volume element. The homogenized elastic properties were analytically estimated using beam-thecry concepts and upper and lower bound techniques as well as using three-dimensional finite element analyses. The homogenized extensional and bending lamina properties are, in general, distinct properties and are not simply related to each other as in monolithic beams and plates or in composites with very fine and highly periodic microstructures. The importance and cause of distinct homogenized extensional and flexural elastic properties is briefly discussed.
\end{abstract}

\subsection{Introduction}

Recently the use of large-tow braided composites has been evaluated by the automotive industry. These materials are manufactured using automated processes and can be used for complicated structural components. They generally exhibit better toughness properties than analogous unidirectional composite stacking sequences because the braided architecture is more resistant to delamination and in plane deformation. In addition, the large tow characteristic permits the use of fewer laminae. However, the complicated fiber architecture of these large-tow braided structures does not permit the use of traditional homogenization techniques to examine their lamina-level mechanical behavior.

1. Work performed under the auspices of the U.S. Department of Energy by Lawrence Livermore National Laboratory under Contract W-7405-Eng-48. 
That large tow braided composite structures possess distinct flexural and extensional properties is the main concern of this study. Whitcomb et al (1998) observed using finite element analysis that the ratio of the effective flexural modulus to the effective extensional modulus for a two ply plain weave composite structure is less than 0.7 . The ratio increases to 0.95 for eight ply structures. It is believed that this phenomenon is geometrically dependent, and thus it is not unique to these textile composites. Pagano (1974) also noted this discrepancy in the flexural and extensional properties for unidirectional boron-epoxy composite structures of too few layers. The phenomenon has not been extensively explored in the literature because traditional small tow composite laminates, widely used in the aerospace industry, generally contain a large number of lamina whose microstructure is relatively fine.

This study aims to examine the elastic flexural and extensional properties of large-tow braided composites using numerical and analytical methods. The geometry of the braided architecture was modeled using idealizations similar to those presented by Naik (1994) and others (e.g., Vandeurzen et al, 1997) in which the composite was idealized as an array of repeating unit cells. The homogenized elastic properties of the unit cell were determined by finite element analysis (FEA). In addition, an analytical estimate of its properties was performed using beam-theory concepts and pseudo upper and lower bound techniques. The development of both the 3-D finite element (FE) and 1-D laminate beam models and their results are discussed in context of the braided architecture.

The following sections describe the development of both the 3-D FE and 1-D laminate beam models and their results. First, the braided geometry is idealized, and a representative volume element or "unit cell" is established. Second, the FE model is presented including descriptions of the specific braided composite material properties and imposed boundary conditions. Also included is a discussion of how classical plate theory was used to infer the effective elastic properties from the numerical simulations. In the third section, two 1-D laminate beam models are constructed. The "bounding" techniques used to infer the mechanical properties from these models are described along with the results. In the final section, the general conditions which require distinct effective extensional and flexural properties to accurately model a braided lamina or laminate are discussed and contrasted to those for traditional unidirectional composites. 


\subsection{Development of the Representative Volume Element}

An appropriate geometric model of the braided composite was developed to analyze the material's mechanical behavior. First, irregular geometric features of the fiber structure were identified. Then they were simplified to develop a workable geometric model of the composite material. Utilizing the periodic nature of the braid structure, a representative volume element (RVE) was identified from the simplified geometric model. It was used in both the 3-D FEA and the 1-D homogenization analysis. The following subsections describes the physical structure of a braided composite and its simplified geometrical representation.

\subsection{Physical Description of Braided Composite}

Figure 2.1 shows a schematic of the triaxial braid fiber structure (Naik et $a l, 1994)$. The three main braiders are oriented in the $+\theta, 0^{\circ}$, and $-\theta$ direction. They are braided in a $1 \times 1$ pattern with the $\pm \theta$ braiders alternately undulating under and over each axial $\left(0^{\circ}\right)$ braider. The fiber bundles of the large tow composites used by automotive industry typically contain on the order of $10 \mathrm{k}$ filaments.

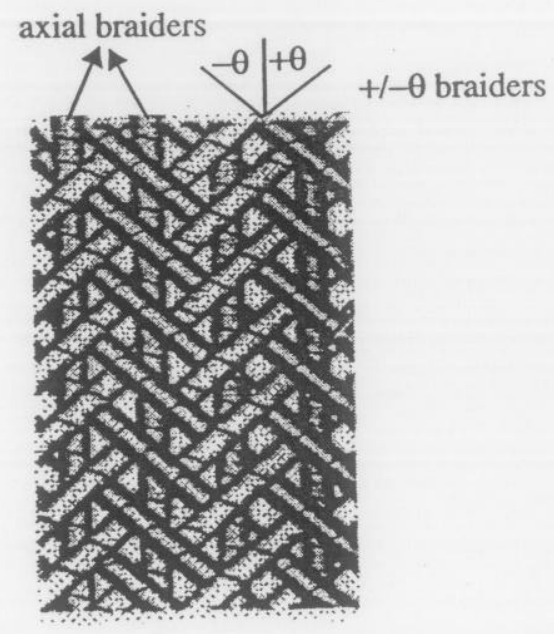

Figure 2.1 Schematic of braid pattern (Naik et al, 1994)

Figure 2.2 shows two sectional micrographs of a multi-ply braided composite laminate (Dadkhah et al, 1995). It can observed that the fiber geometry exhibits many irregular features that are difficult to model. For example only two of the three fiber bundles are in contact typically at the cross over junctions. The junctions are smeared because during manufacturing the braid is allowed to relax causing the braiders to shift. This also causes all three braiders to undulate. The braiding process also causes the initial circular cross section of the braider tow to deform into a lenticular cross section. As a result, the material appears densely packed and contains relatively few pure resin pockets. 


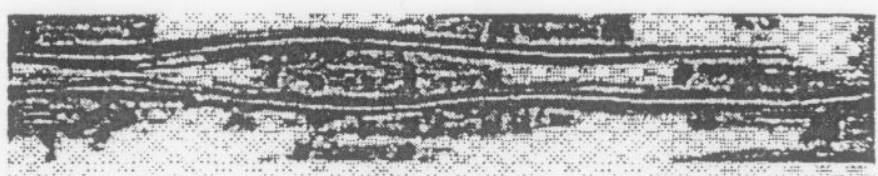

(a)

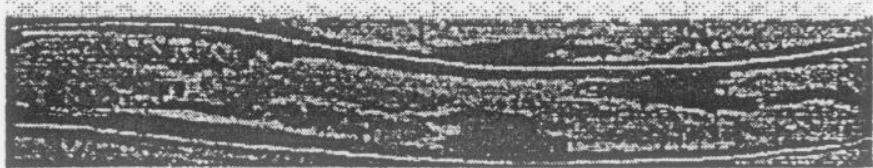

(b)

Figure 2.2 Cross sectional micrographs of a $\left[0^{\circ} \% 40^{\circ}\right], V_{f}=37 \%$, laminate in (a) axial fiber direction, and (b) in braider direction (Dadkhah et al, 1995)

\subsection{Simplified Geometric Model}

A simplified geometric model of the triaxial braided architecture was developed, and is shown in Figure 2.3. All three braiders in the model were assumed to stack up at the cross over regions. This required that the diameter of the axial braider, $d_{a}$, be larger than that of the $\pm \theta$, braiders $d_{b}$. Their ratio was:

$$
\frac{d_{b}}{d_{a}}=\cos \theta
$$

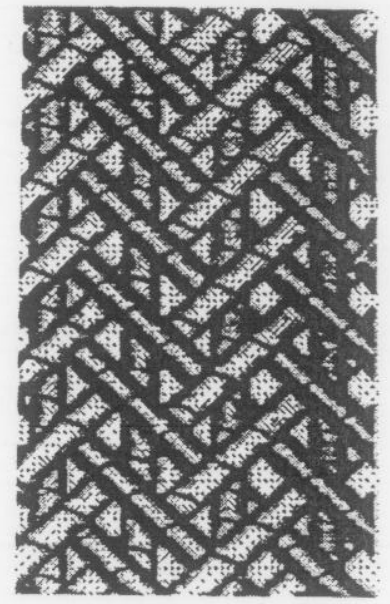

(c)

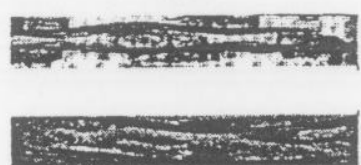

(d)
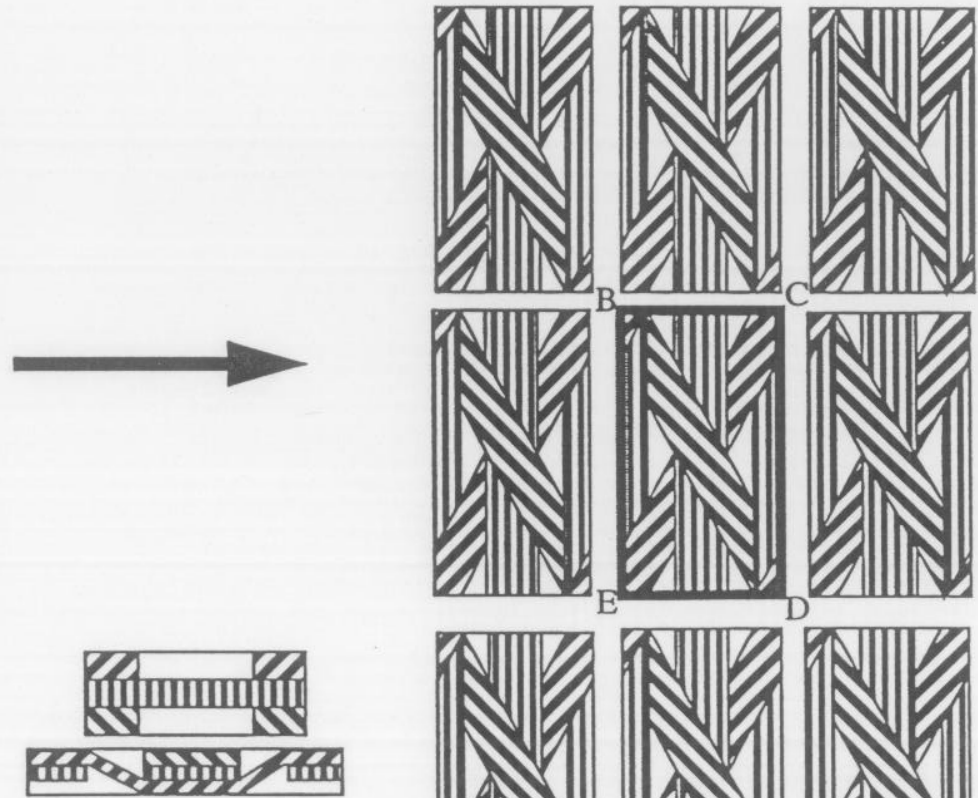

(b)
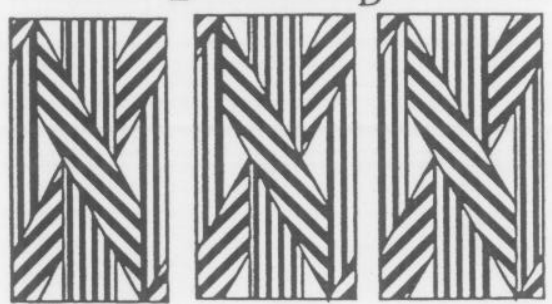

(a)

Figure 2.3 (a) Planar view of geometric model, (b) cross sections of the geometric model along the axial and $-\theta$ braiders, (c) schematic of fiber architecture (Naik et $a l, 1994)$, and (d) cross sectional micrographs along the axial and $\pm \theta$ braiders (Dadkhah $e t$ al, 1995). 
Also, the model only incorporated the undulation of the $\pm \theta$ braiders, and the lenticular cross section of the braiders were simplified to be rectangular to preserve the flattened and expansive characteristic of the fiber tows.

Figure 2.3 shows that the simplified geometric model contains some rather artificial features. Mainly, numerous distinct regions of pure resin and fiber are present causing the model to appear sparse and discontinuous. The transitions from planar fibers to undulated fibers to matrix are abrupt. This is opposed to the dense melded appearance of the sectional micrographs shown in Figure 2.2. These artificial features may generate a softer elastic response in the geometric model compared to that of the actual composite.

\subsection{Geometry of the Representative Volume Element}

The periodic nature of the simplified geometric model permits the repeating unit cell, shown in Figure 2.4, to be chosen as the RVE of the braided composite. The unit cell can be easily identified in Figure 2.3 as the rectangle, BCDE.

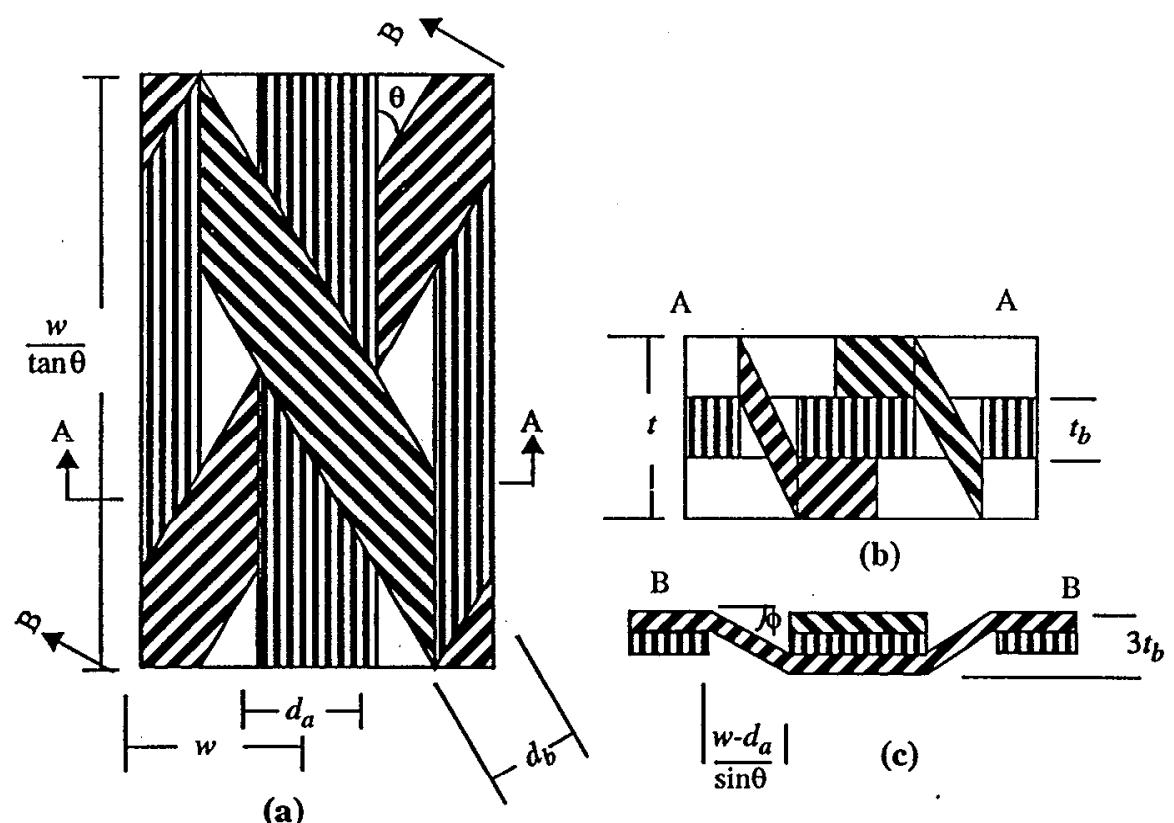

Figure 2.4 The unit cell (a) planar view, (b) cross section in axial braider direction, and (c) cross section in $+\theta$ braider direction

The spacing between the axial braiders, $w$, the braider angle, $\theta$, the filament count, $n$, the filament diameter, $d_{f}$ and the lamina thickness, $t$, were assumed to be manufactured specified quantities. The diameter of the axial braider, $d_{a}$, expressed in Eq. (2) was determined from $d_{f}, n, t$ and the packing density of the axial tows, $p_{a}$. Equation (2) reflects both the initial circular and final flattened geometry of the fiber tows. The braider thickness, $t_{b}$, was set at one third of the lamina thickness. The crimp angle, $\phi$, the undulation angle of the $\pm \theta$ braiders, was determined by Eq. (3) 


$$
\begin{gathered}
d_{a} t=\frac{\left(\frac{\pi}{4} n d_{f}^{2}\right)}{p_{a}} \\
\phi=\operatorname{atan}\left(\frac{2 t_{b}}{\left(w-d_{a}\right) / \sin \theta}\right)
\end{gathered}
$$




\subsection{Finite Element Analysis}

A 3-D FE model of the unit cell was developed to probe the in-plane extensional and flexural elastic properties of the braided composite plate. The FE analysis was conducted using NIKE3D (Maker et al, 1995), a nonlinear implicit FE code developed at LLNL. The following sections discuss the development of the FE model, including the construction of appropriate boundary conditions and loading geometries. The results of the FE analysis are presented in Section 3.5.

\subsection{The Finite Element Mesh}

Figure 3.1a displays a planar view of the 3-D FE mesh. A view of just the braid architecture is shown in Figure 3.1b. The mesh layout was chosen to minimize mesh discontinuities and irregularly shaped elements. These features artificially stiffen the FE model. In the plane, the mesh does not exhibit any irregular features. However, mesh discontinuities and wedge elements were needed to connect the undulations of the $\pm \theta$ braiders to the adjacent resin regions. Mesh discontinuities were resolved using "tied" slide surfaces. The mesh was constructed from 8-node, selectively reduced, incompatible mode, hexahedral elements. Where bending and other higher order deformation modes are present, the incompatible mode formulation results in a more accurate response.

The dimensions of the mesh were chosen to match a specific carbon-fiber material of fiber volume fraction, $V_{f}=30.5 \%$. The axial spacing, $w$, the lamina thickness, $t$, and braid angle, $\theta$, were specified at $1.016 \mathrm{~cm}(0.40 \mathrm{in}), 0.1415 \mathrm{~cm}\left(0.0557 \mathrm{in}\right.$.), and $30^{\circ}$. Each braider consisted of 50k filaments, each with a filament diameter of $d_{f}=7.2 \mu \mathrm{m}$. A moderate packing volume fraction, $p_{a}$, of $70 \%$ was assumed for the axial braiders. Using Eq. (2) the axial fiber diameter, $d_{a}$, was calculated to be $7.112 \mathrm{~mm}(0.28 \mathrm{in}$.). From this, the $\pm \theta$ braider diameter, $d_{b}$, was determined from Eq. (1) to be $8.212 \mathrm{~mm}(0.323 \mathrm{in}$.). This gives the off axis braiders a packing efficiency, $p_{b}$, of $60 \%$ to achieve the necessary $V_{f}$ The crimp angle, $\phi$, was calculated from Eq. (3) to be $8.79^{\circ}$. 


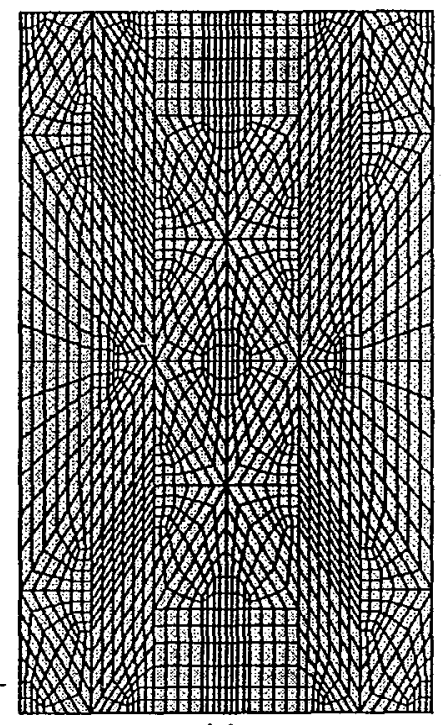

(a)

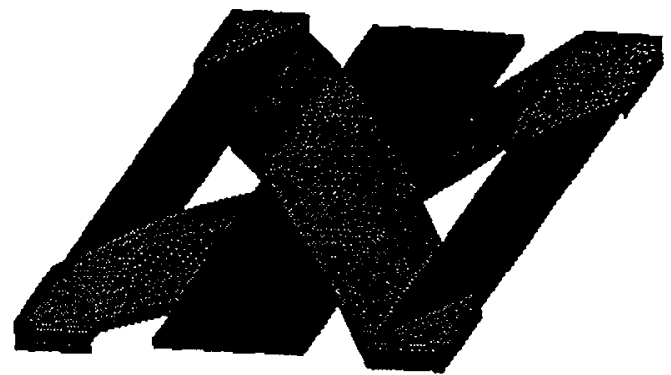

$L^{r} x$

(b)

Figure 3.1 (a) The FE mesh, and (b) fiber structure of the FE mesh

\subsection{Material Properties}

The materials properties used in the FEA were obtained from Marrey and Sankar (1997) for a low modulus graphite-epoxy composite of $V_{f}=64 \%$. The material properties for the $60 \%$ ( $\pm \theta$ braiders) and $70 \%$ (axial braiders) $V_{f}$ regions were obtained by scaling the $V_{f}=64 \%$ data using the rule of mixtures. Table 1 lists their properties. The subscripts 1 and 2 denote fiber and transverse material directions.

TABLE 1. Material Properties of FE model

\begin{tabular}{lrrrrl}
\hline & $E_{1}(\mathbf{G P a})$ & $E_{2}(\mathbf{G P a})$ & $G_{12}(\mathbf{G P a})$ & \multicolumn{1}{l}{$v_{\boldsymbol{l}}$} & \multicolumn{1}{c}{$v_{t t}$} \\
Axial fiber region & 158.375 & 12.830 & 6.038 & 0.230 & 0.300 \\
$\pm \theta$ fiber region & 135.750 & 10.997 & 5.175 & 0.230 & 0.300 \\
Resin region & 3.450 & 3.450 & 3.450 & 0.350 & 0.350
\end{tabular}

\subsection{Boundary Constraints}

Once the geometry of the unit cell was determined, periodic displacement boundary conditions were developed to model the constraints imposed by neighboring unit cells. In accordance with a plane-stress analysis, boundary constraints were applied only on the $x= \pm w$ and the $y= \pm l$ boundaries, where $l=w / \tan \theta$. Two sets of boundary conditions were developed for: (1) in-plane tensile and bending loads, and (2) in-plane shear loads. 


\subsubsection{Tensile and Flexural Model}

The following periodic displacement boundary conditions, $u_{i}(x, y, z)$, were used to model the constraints imposed on the FE mesh boundaries by neighboring unit cells. The constraints maintain a continuous displacement field between adjacent unit cells.

$$
\begin{aligned}
& u_{x}(x, y=l, z)=u_{x}(x, y=-l, z) \\
& u_{y}(x=w, y, z)=u_{y}(x=-w, y, z) \\
& u_{z}(x, y=l, z)=u_{z}(x, y=-l, z) \\
& u_{z}(x=w, y, z)=u_{z}(x=-w, y, z)
\end{aligned}
$$

The center node of the mesh was pinned to lock out various rigid body modes.

$$
u_{x}(x=0, y=0, z=0)=u_{y}(x=0, y=0, z=0)=u_{z}(x=0, y=0, z=0)=0
$$

In addition, as a first order approximation, the $x= \pm w$ and the $y= \pm l$ planes were required to remain planar during deformation. To accomplish this, the cell edges were affixed onto rigid planar shells using "sliding only" slide surfaces. These "sliding only" interfaces permit the mesh boundaries to develop independent tangential displacements along the boundary-shell interface while still constraining the mesh boundaries to reside in the plane of the rigid shells. Consequently, the mesh boundaries transmit no tangential tractions across the interface.

As will be discussed in Section 3.4, classical plate theory was used to evaluate the elastic properties of the unit cell. The coefficients of the extensional, coupling, and bending stiffness matrices were determined by performing a series of simulations with different displacement and rotation boundary conditions imposed on the rigid boundary shells. Each boundary shell has three rotational and three displacement degrees of freedom. To solve for the tensile and flexural terms, the following displacement, $\Delta_{i}$, and rotation, $\Theta_{i}$, boundary conditions were individually imposed on the four rigid shells. The terms, $\varepsilon_{11}$ and $\varepsilon_{22}$ are the desired nominal strain in the transverse and longitudinal direction, respectively, and $\kappa_{11}$ and $\kappa_{22}$ are the desired nominal curvature about the transverse and longitudinal axes. The loading geometries are illustrated in Figure 3.2.

$$
\begin{gathered}
\Delta_{x}(x=w)=-\Delta_{x}(x=-w)=\varepsilon_{11} w \quad\left(\text { all other } \Delta_{i} \text { and } \Theta_{i} \text { zero }\right) \\
\Delta_{y}(y=l)=-\Delta_{y}(y=-l)=\varepsilon_{22} l \quad\left(\text { all other } \Delta_{i} \text { and } \Theta_{i} \text { zero }\right) \\
\Theta_{x}(y=l)=-\Theta_{x}(y=-l)=\kappa_{22} l\left(\text { all other } \Delta_{i} \text { and } \Theta_{i} \text { zero }\right) \\
\Theta_{y}(x=w)=-\Theta_{y}(x=-w)=\kappa_{11} w\left(\text { all other } \Delta_{i} \text { and } \Theta_{i} \text { zero }\right)
\end{gathered}
$$




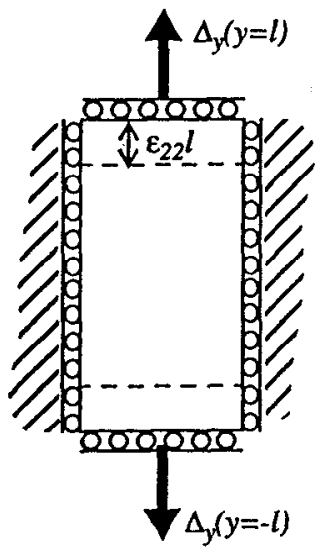

(a)

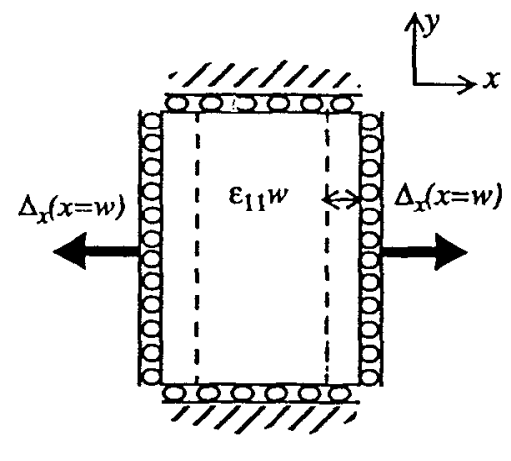

(b)

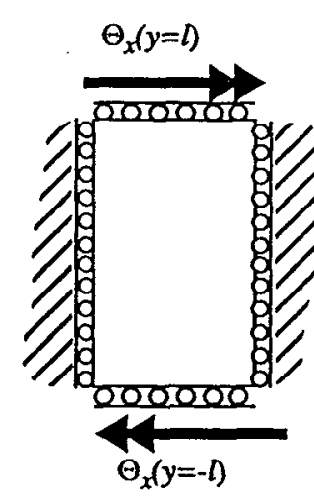

(c)

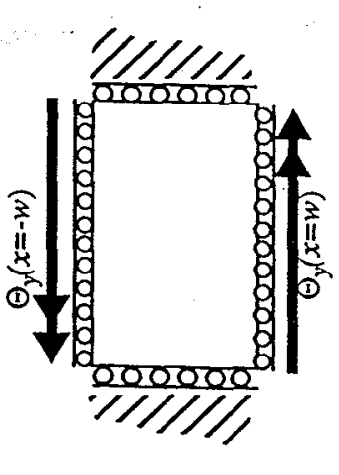

(d)

Figure 3.2 Illustration of loading geometry for (a) tension in Iongitudinal direction, (b) tension in the transverse direction, (c) rotation about the transverse axis, and (d) rotation about longitudinal axis

The applied displacements and rotations were small so that the resulting strains and curvatures developed in unit cell were on the order of $10^{-5}$. The small values were chosen to avoid non-linear geometry affects.

\subsubsection{Shear Model}

To generate pure in-plane shear, "tied" slide surfaces were used to affix the mesh boundaries onto the rigid boundary shells. The "tied" interfaces restrict all independent tangential and normal displacements at the mesh boundary-shell interface. As a result, tangential loads can be transmitted to the mesh through the "tied" sliding surfaces. Another set of displacement and rotation loading boundary constraints were applied to the "tied" rigid shells to determine the shear terms in the $[A],[B]$, and $[D]$ matrices. The constraints expressed by Eq. (13)-(14) were individually imposed to determine the inplane shear modulus and the shear-extension coupling terms.

$$
\begin{gathered}
\Delta_{x}(y=l)=\gamma_{21} l \\
\Delta_{x}(y=-l)=\theta_{z}(y= \pm l)=0 \\
\Delta_{y}=\Delta_{z}=\theta_{x}=\theta_{y}=0, \text { for all boundary shells } \\
\Delta_{y}(x=w)=\gamma_{12} w \\
\Delta_{y}(x=-w)=\theta_{z}(x= \pm w)=0 \\
\Delta_{z}=\theta_{x}=\theta_{y}=0, \text { for all boundary shells }
\end{gathered}
$$




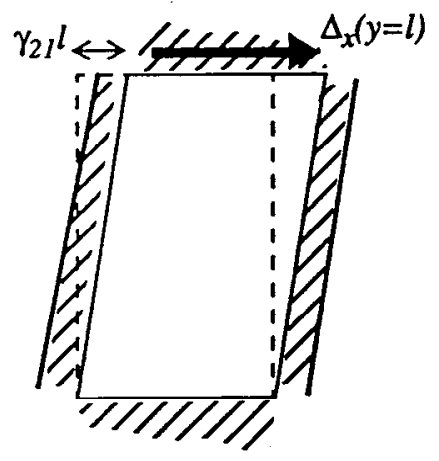

(a)

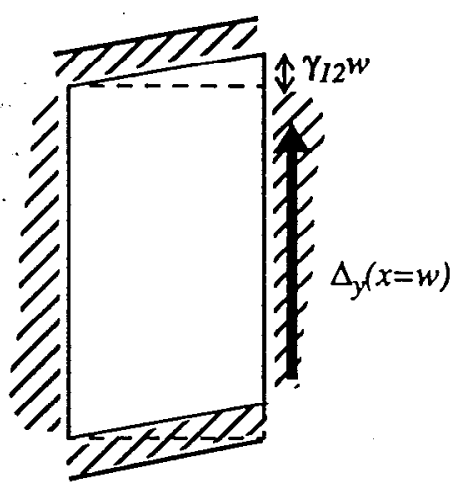

(b)

Figure 3.3 Illustration of loading geometry for (a) shearing in the transverse direction, and (b) shearing in the longitudinal direction.

\subsection{Determining the In-Plane Elastic Constants}

As described in the previous section, selective displacements and rotations were applied to the boundary shells of the FE model to probe its elastic response. Classical plate theory was used to infer the elastic properties of the unit cell from the reaction forces obtained at the mesh boundary-shell interfaces. Equation (15) shows the constitutive model for plates, in which the [A], [B], and [D] matrices relate $N_{i j}$ and $M_{i j}$, the force per unit length and moment per unit length, to $\varepsilon^{0}{ }_{i j}$ and $\kappa_{i j}^{0}$, the midplane strains and curvatures.

$$
\left[\begin{array}{l}
N_{11} \\
N_{22} \\
N_{66} \\
M_{11} \\
M_{22}
\end{array}\right]=\left[\begin{array}{lllll}
A_{11} & A_{12} & A_{16} & B_{11} & B_{12} \\
A_{21} & A_{22} & A_{26} & B_{21} & B_{22} \\
A_{61} & A_{62} & A_{66} & B_{61} & B_{62} \\
B_{11} & B_{21} & B_{62} & D_{11} & D_{12} \\
B_{12} & B_{22} & B_{62} & D_{21} & D_{22}
\end{array}\right]\left[\begin{array}{c}
\varepsilon_{11}^{o} \\
\varepsilon_{22}^{o} \\
\gamma_{66}^{o} \\
\kappa_{11}^{o} \\
\kappa_{22}^{o}
\end{array}\right]
$$

Eq. (16) relates the average plate stress, $\sigma^{*}$, and the average moments, $\left(\frac{z}{t} \sigma\right)^{*}$, to the resultant forces and moments; 
The resultant forces and moments were obtained from the reaction forces and moments, $F_{x}, F_{y}, M_{x}$ and $M_{y}$ of the rigid shells as shown below.

$$
\left[\begin{array}{c}
N_{11} \\
N_{22} \\
N_{66} \\
M_{11} \\
M_{22}
\end{array}\right]=\left[\begin{array}{c}
F_{x} /(2 l) \\
F_{y} /(2 w) \\
F_{x} /(2 w) \\
M_{y} /(2 l) \\
M_{x} /(2 w)
\end{array}\right]
$$

Since only one non-zero midplane strain or curvature was imposed in each FEA, the resultant forces and moments, when normalized by the imposed strain or curvature, directly correspond to the coefficients of the [A], [B], and [D] matrices. Finally the inplane homogenized extensional and flexural Young's moduli, $E_{1}, E_{2}, E_{f l}$, and $E_{f 2}$, shear modulus, $G$, and Poisson's ratios, $v_{12}$, and, $v_{f 12}$, of the unit cell were obtained by inverting the $[A]$ and $[D]$ matrices as shown in Eq.(18) and (19). The terms, $a_{i 6}$ and $a_{6 j}$ are the inplane shear-extensional coupling terms.

$$
\begin{aligned}
& {\left[\begin{array}{ccc}
\frac{1}{E_{1}} & \frac{-v_{12}}{E_{1}} & a_{16} \\
\frac{-v_{21}}{E_{2}} & \frac{1}{E_{2}} & a_{26} \\
a_{61} & a_{62} & \frac{1}{G}
\end{array}\right]=[A]^{-1} t} \\
& {\left[\begin{array}{cc}
\frac{1}{E_{f 1}} & \frac{-v_{f 12}}{E_{f 1}} \\
\frac{-v_{f 21}}{E_{f 2}} & \frac{1}{E_{f 2}}
\end{array}\right]=[D]^{-1} \frac{t^{3}}{12}}
\end{aligned}
$$

\subsection{FE Results}

As a check, the FE model was used to calculate the in-plane extensional, shear, and flexural properties of a homogenous isotropic unit cell. The results were accurate to within $+-0.1 \%$. The following subsections present the results of the FE analysis for the braided composite unit cell. First, the flexural and extensional properties of the unit cell are presented and compared. Then, the values of the bending-extensional coupling matrix and the shear-extension coupling terms are discussed.

\subsubsection{Comparison of Flexural and Extensional Moduli}

Table 2 presents the in-plane extensional and flexural Young's moduli and Poisson's ratios of the braided composite unit cell as determined for imposed strains of $10^{-5}$ and rotations of $5 \times 10^{-6}$. The results show a large difference between the in-plane extensional and 
flexural moduli. In the longitudinal direction, the flexural modulus was a mere $32 \%$ of the extensional modulus. Similarly, in the transverse direction, the flexural modulus was $71 \%$ of the extensional modulus. This dramatic difference in the properties can be attributed to the fiber architecture of the unit cell.

TABLE 2. Elastic Properties of the Unit Cell as Determine by FEA

\begin{tabular}{lrrrl}
\hline & $\mathbf{E}_{\mathbf{l}}(\mathbf{G P a})$ & $\mathbf{E}_{\mathbf{t}}(\mathbf{G P a})$ & $\mathbf{G}_{\mathbf{l t}}(\mathbf{G P a})$ & \multicolumn{1}{l}{$\mathbf{v}_{\mathbf{l t}}$} \\
Extensional & 51.926 & 7.646 & 6.523 & 0.766 \\
Flexural & 16.610 & 5.419 & $\ldots$ & 0.589
\end{tabular}

Figure 3.4 shows the bending stress contour of the unit cell subjected to a rotation about the transverse axis. The resin material has been removed and the displacements have been magnified by $10^{5}$ to better observe the behavior of the braiders. The contours show that the bending stress experienced by the off axis braiders is approximately twice that of the axial braiders. The \pm 30 braiders support significantly higher bending loads because they lie farther away from the neutral axis as compared to the axial braiders whose midplane lies on the neutral plane. Consequently, only the \pm 30 braiders contribute significantly to the overall bending stiffness of the unit cell.

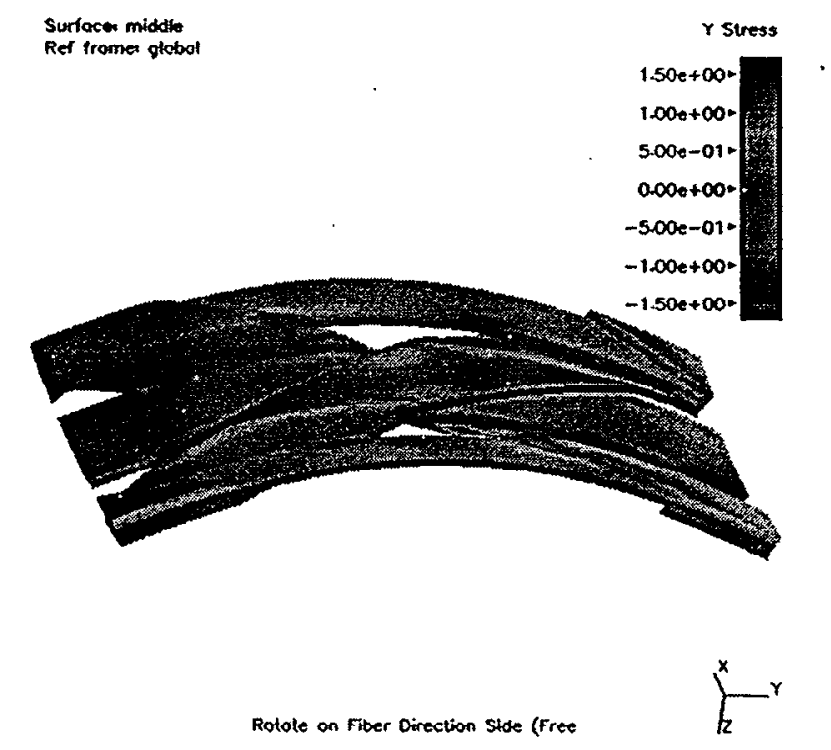

Figure 3.4 Bending stress contour of unit cell subjected to a rotation about the transverse axis

Unlike in the bending case, all three braidcrs significantly contribute to the overall extensional stiffness of the unit cell. Figure 3.5 shows the tensile stress contours of the unit cell subjected to a longitudinal strain. A significant reduction in the longitudinal stress is observed at the crossover region. This indicates that the load is being effectively distributed among the three braiders. The axial fiber $\left(E_{1}=158 \mathrm{GPa}\right)$ is significantly stiffer than the rotated \pm 30 braiders $\left(E_{1}=25 \mathrm{GPa}\right)$ in the longitudinal direction. Consequently, the overall extensional stiffness, dependent on the stiffness of both the axial and \pm 30 braiders, is significantly higher than the overall bending stiffness, which depends primarily on the stiffness of the \pm 30 braiders. 


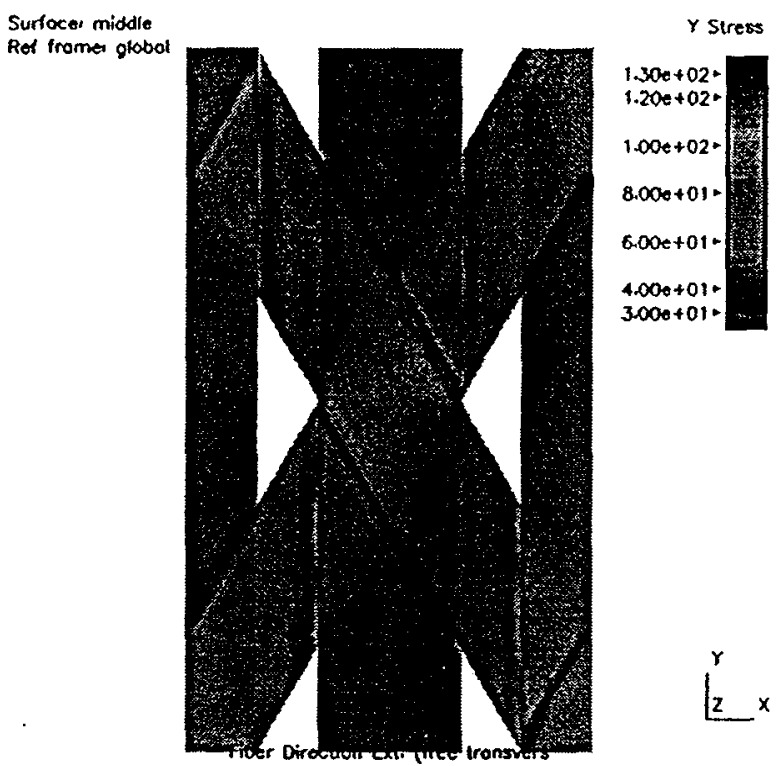

Figure 3.5 Longitudinal tensile stress contour of unit cell subjected to longitudinal strain

\subsubsection{The Bending-Extension and Shear-Extension Coupling Terms}

FEA was also used to determine the terms of the bending-extension coupling stiffness matrix and the shear-extension coupling terms. In general, the values were several orders of magnitude smaller than the terms in the bending and extensional matrices, and many of the coefficients demonstrated a strong dependence upon the magnitude of the imposed deformation - a behavior attributed to non-linear geometric affects. Consequently, the coupling stiffness coefficients were approximated to be zero. It is proposed that no significant coupling between the bending and extensional response and between the shear and extension response of the unit cell was observed because of the symmetric nature of the braid architecture. The distributions of the $\pm \theta$ planar braider materials and the $\pm \theta / \pm \phi$ undulating braider materials are symmetric about the midpoint and midplane of the unit cell. 


\subsection{Homogenized Beam Analysis}

Two simple 1-D Bernoulli-like beam models of the unit cell, one with the neutral axis in the longitudinal direction and the other in the transverse direction, were developed to evaluate its in-plane elastic properties. For both directions, classical lamination theory was used to determine the effective $[A],[B]$ and $[D]$ matrices for each cross section. From this, the effective extensional and flexural Young's moduli, $E^{*}$ and $E_{f}{ }^{*}$, as a function of distance along the neutral axis were determined. Finally, applying an elementary form of variational analysis, pseudo upper and lower bound estimates of the average effective extensional and flexural Young's moduli, $\left\langle E^{*}\right\rangle$ and $\left\langle E_{f}^{*}\right\rangle$ of the unit cell were inferred from the cross sectional $[A]$ and $[D]$ matrices.

The following subsections discuss the analytical procedure in more detail including: (1) developing the geometry of the beam models, (2) determining the constitutive properties of each material region, (3) calculating the $[\mathrm{A}],[\mathrm{B}]$, and $[\mathrm{D}]$ matrices for each cross section, and (4) evaluating upper and lower bound estimates of the homogenized extensional and flexural Young's moduli. The analytical and FE results are presented in Section 4.5. Though the 3-D FE based results are more accurate, the simple 1-D beam models yield insight to how the homogenized material properties depend on the braid architecture.

\subsection{Geometry of Beam Models}

Figure 4.1 and Figure 4.2 show two 1-D beam models of the unit cell, the former with the neutral axis in the longitudinal direction, and the latter with the neutral axis in the transverse direction. The beams consist of three equal thickness plies of inhomogenous material. Consistent with the Bernoulli beam theory and lamination theory, planar sections were assumed to remain planar after deformation.

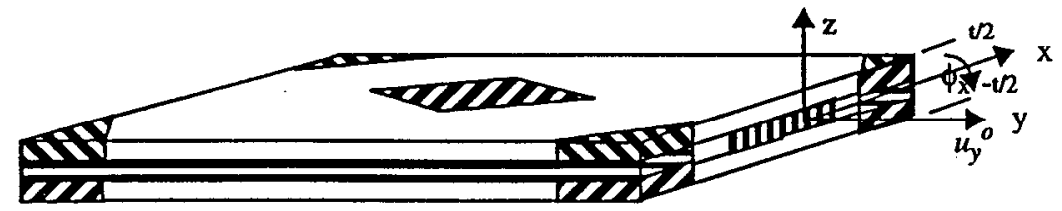

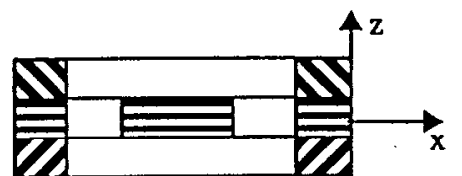

(b)

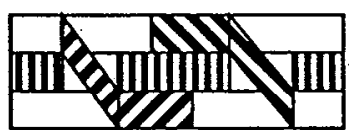

(c)

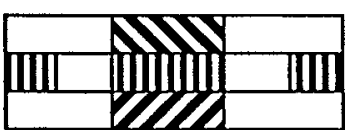

(d)

Figure 4.1(a) 1-D longitudinal beam model of unit cell, (b) cross section at $y=-l$, (c) cross section at $y=-\left(l-\frac{d a}{\tan \theta}\right)$, and (d) cross section at $y=0$ 


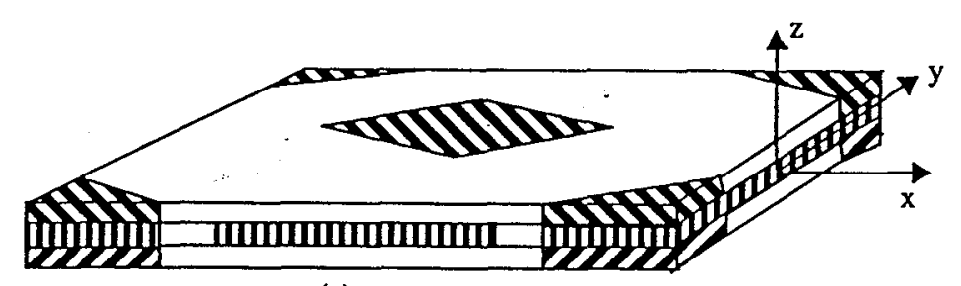

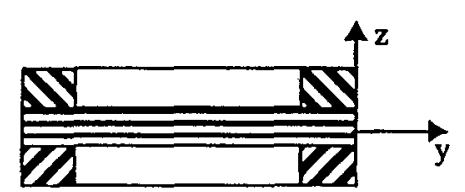

(b)

(a)

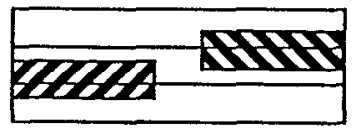

(c)

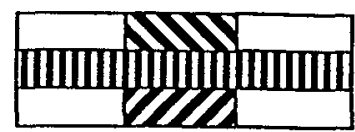

(d)

Figure 4.2 (a) 1-D transverse beam model of unit cell, (b) cross section at $x=w$, (c) cross section at $x=w / 2$, and (d) cross section at $x=0$

\subsection{Material Region Properties}

The stiffness matrices of the resin, axial braiders, $\pm \theta$ planar braiders, and $\pm \theta / \pm \phi$ undulating braiders, must be expressed in the global coordinate system before the average properties of the unit cell can be determined. First, the $6 \times 6$ orthotropic stiffness matrix, [C], expressed in the local material coordinate systems, was determined for the resin and braiders directly from the Young's moduli and Poisson's ratios listed in Table 1. Then the [C] matrices were transformed into the global coordinate system using the transformation matrix, [T], as shown in Eq. (20).

$$
[Q]=[T]^{-1}[C][T]
$$

The matrix [T] is formed from the direction cosines between the material coordinates and the global coordinates. The material directions of the $\pm \theta$ planar braiders, and $\pm \theta / \pm \phi$ undulating braiders are expressed in Eq. (21) and (22), respectively. The material directions of the axial braiders are coincident with the global coordinate system, and the resin is isotropic. Therefore, the transformation was applied only to the $\pm \theta$ planar braiders, and $\pm \theta / \pm \phi$ undulating braiders.

$$
\begin{gathered}
{\left[\begin{array}{l}
e_{1} \\
e_{2} \\
e_{2}
\end{array}\right]=\left[\begin{array}{ccc}
\cos \theta & \sin \theta & 0 \\
\sin \theta & \cos \theta & 0 \\
0 & 0 & 1
\end{array}\right]\left[\begin{array}{l}
e_{x} \\
e_{y} \\
e_{z}
\end{array}\right]} \\
{\left[\begin{array}{l}
e_{1} \\
e_{2} \\
e_{2}
\end{array}\right]=\left[\begin{array}{ccc}
\cos \theta & \sin \theta & 0 \\
-\cos \phi \sin \theta & \cos \phi \cos \theta & \sin \phi \\
\sin \phi \sin \theta & \cos \phi \cos \theta & \cos \phi
\end{array}\right]\left[\begin{array}{l}
e_{x} \\
e_{y} \\
e_{z}
\end{array}\right]}
\end{gathered}
$$

The [Q] matrix is a full $6 \times 6$ anisotropic constitutive matrix. Enforcing a plane-stress condition reduces $[\mathrm{Q}]$ to the laminate constitutive matrix, $[\bar{Q}]$, expressed in Eq. (23). The 
$j=1,2$ terms refer to the in-plane extensional properties while the $j=6$ terms pertain to the in-plane shear properties.

$$
[\bar{Q}]=\left[\begin{array}{lll}
Q_{11} & Q_{12} & Q_{16} \\
Q_{12} & Q_{22} & Q_{26} \\
Q_{16} & Q_{26} & Q_{66}
\end{array}\right]
$$

\subsection{Calculating the Effective Cross-Sectional Flexural and Extensional Stiffness}

The [A], [B], and [D] matrices were evaluated for each material region by integrating the stiffness matrix in the through thickness direction, $z$, as shown below.

$$
\begin{gathered}
{[A]=\int_{-t / 2}^{t / 2}[\bar{Q}] d z=\sum_{k=1}^{3} \int_{z_{k-1}}^{z_{k}}[\bar{Q}] d z} \\
{[B]=\int_{-t / 2}^{t / 2}[\bar{Q}] z d z=\sum_{k=1}^{3} \int_{z_{k-1}}^{z_{k}}[\bar{Q}] z d z} \\
{[D]=\int_{-t / 2}^{t / 2}[\bar{Q}] z^{2} d z=\sum_{k=1}^{3} \int_{z_{k-1}}^{z_{k}}[\bar{Q}] z^{2} d z}
\end{gathered}
$$

Because planar sections are idealized to remain plane during deformation, the effective $\left[A^{*}\right],\left[B^{*}\right]$, and $\left[D^{*}\right]$ matrices can be calculated for each cross section by integrating the $[A],[B]$, and $[D]$ matrices along the width of the cross section as shown in Eq. (25) and (26). The subscripts $l$ and $t$ denote the longitudinal and transverse orientation of the neutral axis

$$
\begin{aligned}
& {\left[A^{*}\right]_{l}=\int_{-w}^{w}[A] d x} \\
& {\left[B^{*}\right]_{l}=\int_{-w}^{w}[B] d x} \\
& {\left[D^{*}\right]_{l}=\int_{-w}^{w}[D] d x} \\
& {\left[A^{*}\right]_{t}=\int_{-l}^{l}[A] d y} \\
& {\left[B^{*}\right]_{t}=\int_{-l}^{l}[B] d y} \\
& {\left[D^{*}\right]_{t}=\int_{-l}^{l}[D] d y}
\end{aligned}
$$


The effective extensional and flexural moduli as a function of distance along the neutral axis, can be obtained from $\left[\mathrm{A}^{*}\right]$ and $\left[\mathrm{D}^{*}\right]$ as demonstrated in the following section.

\subsection{Upper and Lower Bound Estimates of Homogenized Moduli}

A simple form of variational analysis was employed to calculate pseudo lower and upper bound estimates for the homogenized extensional and flexural Young's moduli from the cross sectional $\left[\mathrm{A}^{*}\right]$ and $\left[\mathrm{D}^{*}\right]$ matrices. The upper bound was obtained by averaging the effective stiffness matrices, $\left[\mathrm{A}^{*}\right]$ and $\left[\mathrm{D}^{*}\right]$, along the neutral axis, as demonstrated by $\mathrm{Eq}$. (27)

$$
\left\langle\left[A^{*}\right]\right\rangle t=\frac{\int_{-l}^{l}\left[A^{*}\right] l d y}{\int_{-l}^{l} d y}
$$

Analogously, the lower bound was obtained by averaging the effective compliance matrices, $\left[a^{*}\right]$ and $\left[d^{*}\right]$, along the neutral axis, as demonstrated by Eq. (28), where

$$
\left\langle\left[a^{*}\right]\right\rangle_{l}=\frac{\int_{-l}^{l}\left[a^{*}\right] l d y}{\int_{-l}^{l} d y}
$$

$\left[\mathrm{a}^{*}\right]=\left[\mathrm{A}^{*}\right]^{-1}$ and $\left[\mathrm{d}^{*}\right]=\left[\mathrm{D}^{*}\right]^{-1}$. From the averaged stiffness and compliance matrices, the homogenized extensional and flexural moduli in the transverse and longitudinal directions were obtained as follows:

$$
\begin{gathered}
{\left[\begin{array}{c}
\left\langle E_{t}^{*}\right\rangle^{u} \\
\left\langle E_{l}^{*}\right\rangle^{u} \\
\left\langle E_{f t}^{*}\right\rangle^{u} \\
\left\langle E_{f l}^{*}\right\rangle^{u}
\end{array}\right]=\left[\begin{array}{c}
\left\langle\left[A_{11}^{*}\right]\right\rangle^{-1} / l t \\
\left\langle\left[A_{22}^{*}\right]\right\rangle_{l}^{-1} / w t \\
\left\langle\left[D_{11}^{*}\right]\right\rangle_{t}^{-1} /\left(\frac{l t^{3}}{12}\right) \\
\left\langle\left[D_{22}^{*}\right]\right\rangle_{l}^{-1} /\left(\frac{w t^{3}}{12}\right)
\end{array}\right]} \\
{\left[\begin{array}{c}
\left\langle E_{t}^{*}\right\rangle^{l} \\
\left\langle E_{l}^{*}\right\rangle^{l} \\
\left\langle E_{f t}^{*}\right\rangle^{l} \\
\left\langle E_{f l}^{*}\right\rangle^{l}
\end{array}\right]=\left[\begin{array}{c}
\left(\left[a_{11}^{*}\right]\right\rangle_{t}^{-1} / l t \\
\left\langle\left[a_{22}^{*}\right]\right\rangle_{l}^{-1} / w t \\
\left\langle\left[d_{11}^{*}\right]\right\rangle_{l}^{-1} /\left(\frac{l t^{3}}{12}\right) \\
\left\langle\left[d_{22}^{*}\right]\right\rangle_{l}^{-1} /\left(\frac{w t^{3}}{12}\right)
\end{array}\right]}
\end{gathered}
$$


The superscripts, $l$ and $u$, refer to lower and upper bound estimates, respectively. Note, these quantities are not true 3-D lower and upper bounds since neither appropriate stress or deformation fields were imposed to evaluate them. However, within the context of a 2D plane-stress theory, the upper bound estimates may be viewed as true upper bounds.

\subsection{Analytical Results}

The geometric and material properties of the unit cell used in the FEA were again used in the homogenization analysis. In the following sections, the pseudo upper and lower bound estimates of the homogenized moduli, and the terms of the bending-extension coupling matrix are presented and compared to the FE results.

\subsubsection{Upper and Lower Bound Estimates of the Homogenized Moduli}

The effective moduli, $E^{*}$ and $E_{f}^{*}$, as a function of distance along the neutral axis are plotted in Figures 4.3 to 4.6. Little variation is observed in the longitudinal effective moduli. The minimum and maximum values of $E_{l}{ }^{*}$ differs only by $0.5 \%$ as compared to the $82 \%$ difference between the minimum and maximum values of $E_{t}{ }_{t}$. Similarly, the minimum and maximum of $E_{f l}{ }^{*}$ differs by $21 \%$ as compared to the $93 \%$ difference for $E_{f t}{ }^{*}$. The cause of the distributions can be understood by cxamining the braid architecture of the unit cell.

As illustrated in Figure 4.1, the cross-sectional areas of the $\pm \theta$ braiders and the axial braider remain constant along the longitudinal direction. Only the cross sectional areas of the $\pm \theta$ planar braiders and the $\pm \theta / \pm \phi$ undulating braiders, the sum of which gives the cross-sectional areas of the $\pm \theta$ braiders, vary along the longitudinal direction. Thus, the distribution observed in the effective longitudinal moduli is the direct result of this material distribution. The variation for the effective moduli is small because of the small difference in stiffness properties between the undulating and planar $\pm \theta$ braiders. This can be attributed to the small crimp angle, $\phi=8.79^{\circ}$,

Similarly, the distribution of the $E_{t}{ }^{*}$ modulus reflects the distribution of the axial braider material as shown in Figure 4.2. The regions of high and low $E_{t}{ }^{*}$ corresponds to the presence and absence of the axial fiber in the transverse cross sections.

The dramatic variation in the $E_{f t}{ }^{*}$ modulus is directly caused by the undulation in the off axis braiders. In the non-undulating regions, the cross-section of the $\pm \theta$ braiders are at their maximum distance from the neutral axis as shown in Figure 4.2a. During undulation, their cross-section moves towards the neutral axis so that at $x= \pm w / 2$ their midplane lies on the neutral surface as shown in Figure 4.2b. Thesc two regions correspond to the regions of minimum and maximum $E_{f t}{ }^{*}$. The axial braiders do not contribute significantly to $E_{f t}{ }^{*}$ or the local variation in $E_{f t}{ }^{*}$ because their midplane always lies on the neutral surface. 


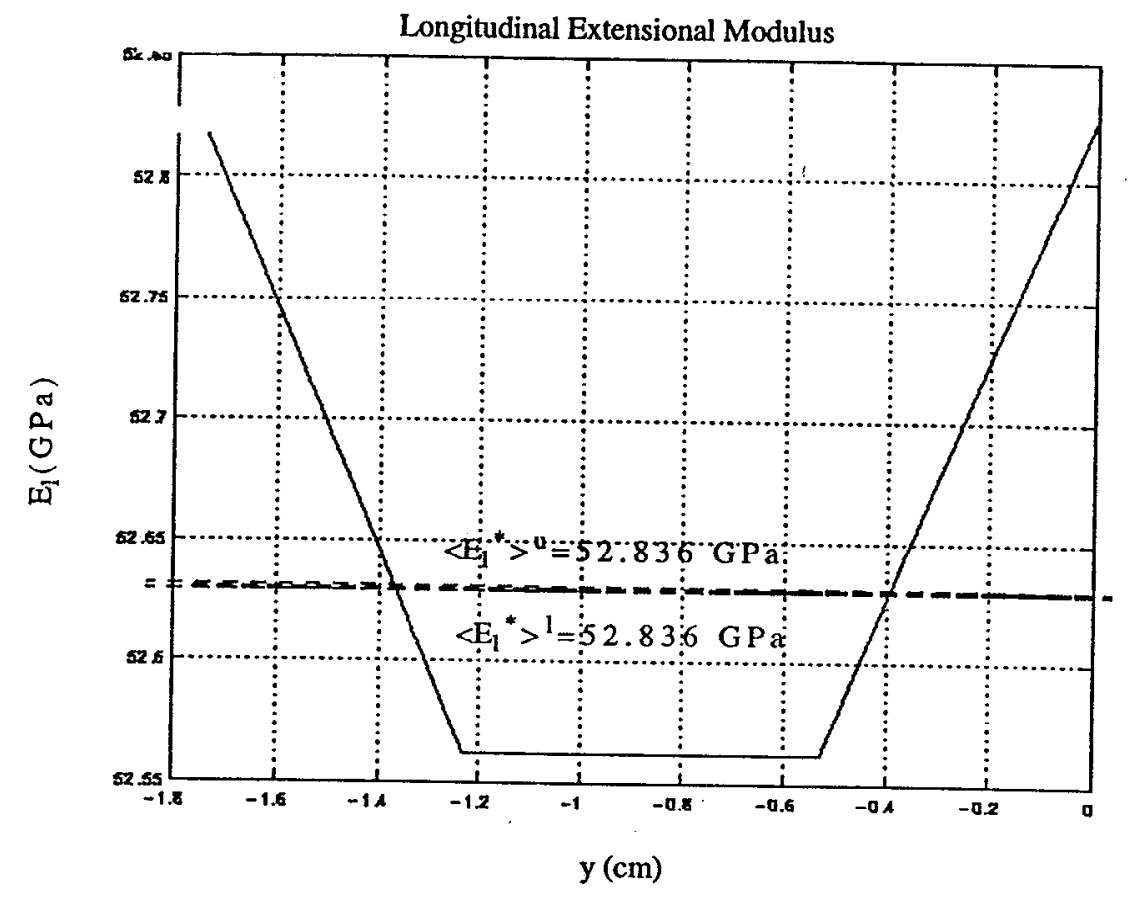

Figure 4.3 Distribution of the effective longitudinal extensional Young's modulus

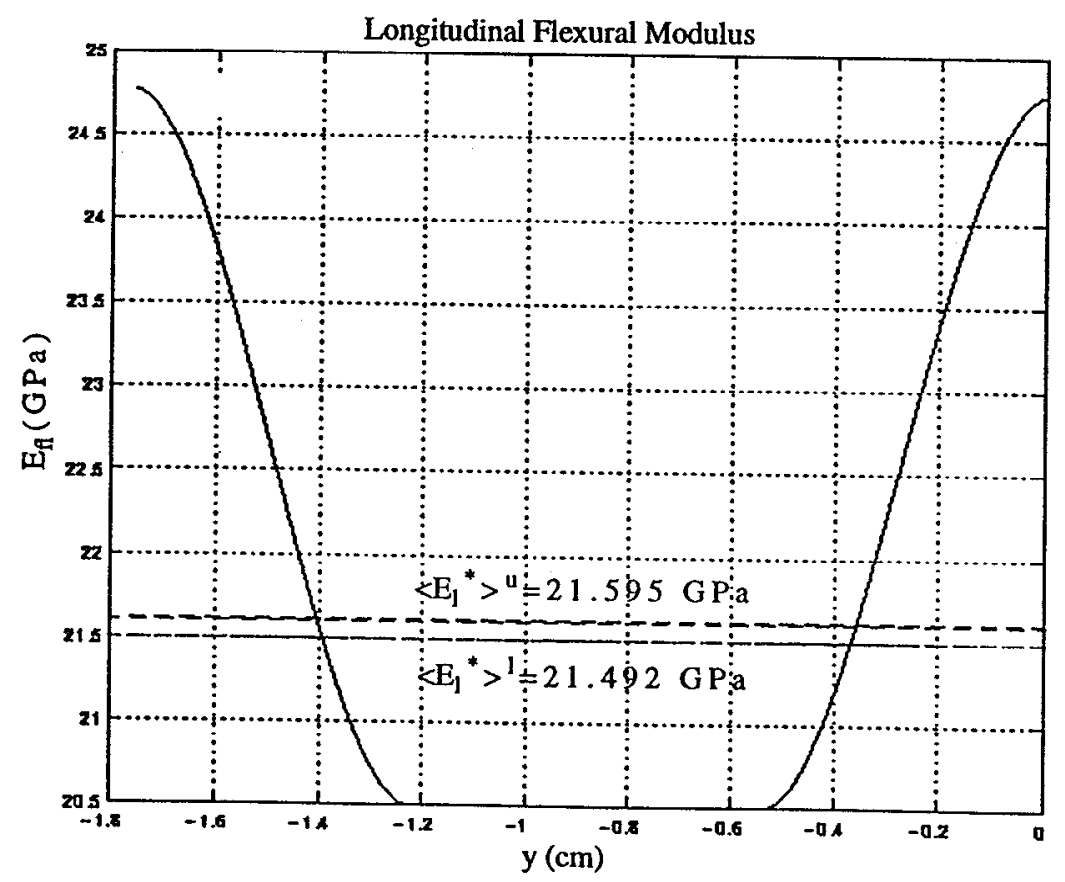

Figure 4.4 Distribution of the effective longitudinal flexural Young's modulus 


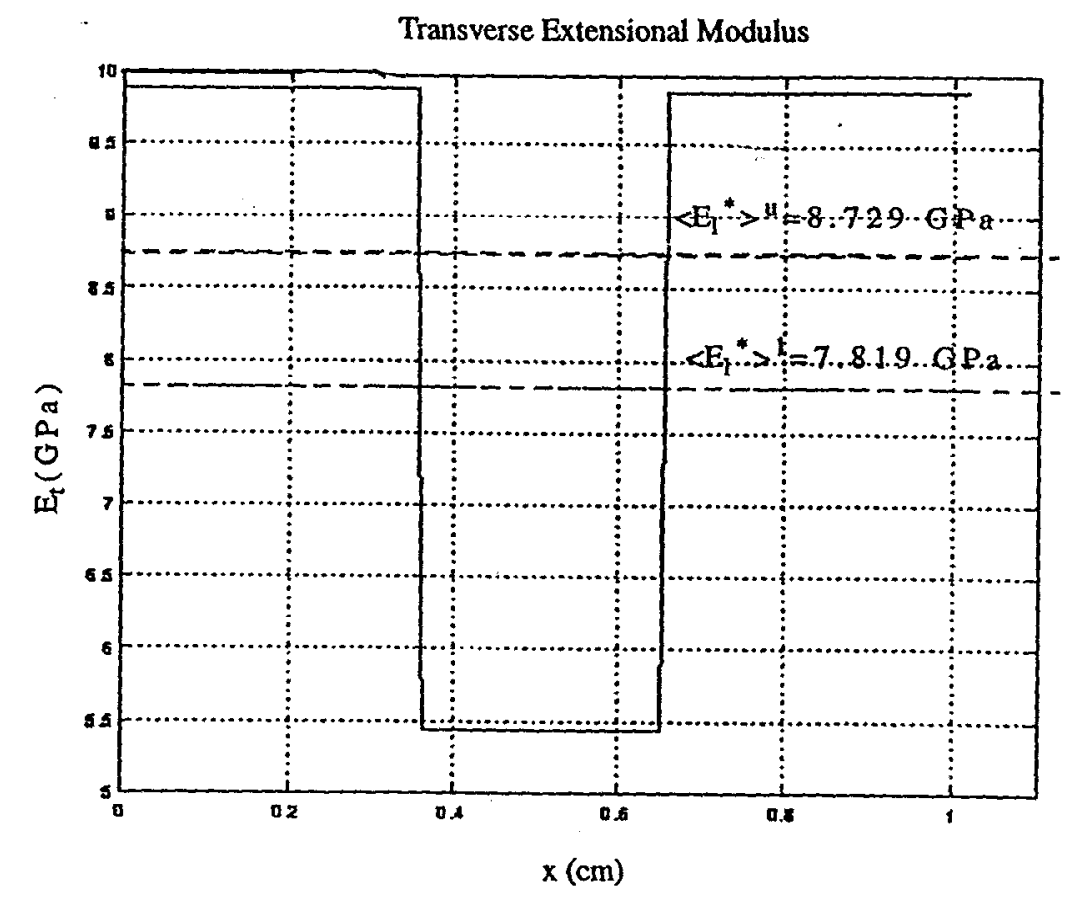

Figure 4.5 Distribution of the effective transverse extensional Young's modulus

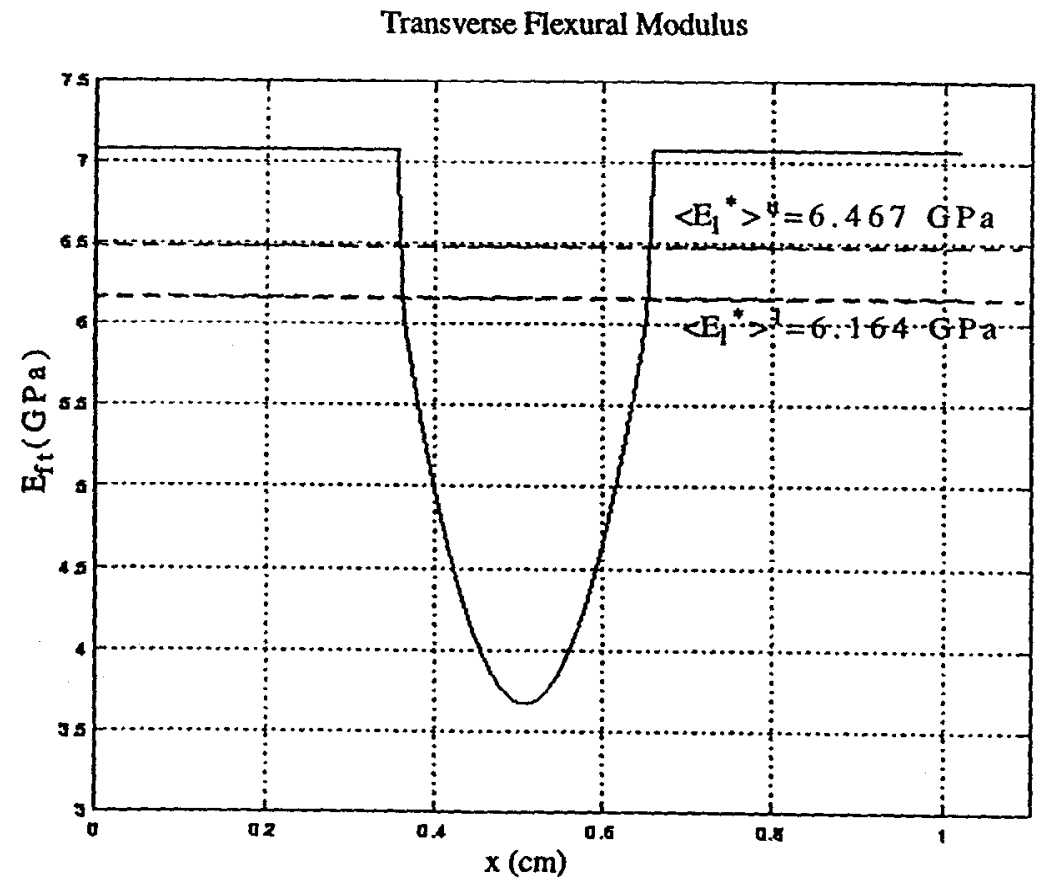

Figure 4.6 Distribution of the effective transverse flexural Young's modulus 
Table 3 presents the upper and lower bound estimates of the homogenized Young's moduli, $\left\langle E^{*}\right\rangle$ and $\left\langle E_{f}^{*}\right\rangle$, and compares them with the $\mathrm{FE}$ results. The estimated homogenized extensional properties are within $15 \%$ of the $\mathrm{FE}$ results. The cstimates for $\left\langle E_{l}^{*}\right\rangle$ and the corresponding FE value only differ by $2 \%$. Consequently, it can be assumed that the homogenization method allows a quick and accurate method to determine overall longitudinal extensional stiffness of the unit cell.

TABLE 3. Upper and Lower Bound Estimates of Homogenized Extensional and Flexural Young's Moduli

\begin{tabular}{lrrrr}
\hline & $\mathbf{E}_{\mathbf{l}}(\mathbf{G P a})$ & $\mathbf{E}_{\mathfrak{t}}(\mathbf{G P a})$ & $\mathbf{E}_{\mathbf{f l}}(\mathbf{G P a})$ & $\mathbf{E}_{\mathrm{ft}}(\mathbf{G P a})$ \\
$\begin{array}{l}\text { Upper Bound Esti- } \\
\text { mates }\end{array}$ & 52.836 & 8.729 & 21.595 & 6.467 \\
$\begin{array}{l}\text { Lower Bound } \\
\text { Estimates }\end{array}$ & 52.836 & 7.819 & 21.492 & 6.164 \\
FE Calculations & 51.926 & 7.646 & 16.610 & 5.419
\end{tabular}

\subsubsection{The Bending-Extension Coupling Terms}

The terms of the bending-extension matrix were calculated to be zero. Coupling between the bending and extensional properties of the unit cell is not present because of the symmetric distributions of the $\pm \theta$ planar braider materials and the $\pm \theta / \pm \phi$ undulating braider materials about the midplane. 


\subsection{Summary and Discussion}

A unit cell model of the braided composite was developed. The elastic extensional and flexural properties of the cell were determined by two techniques: (1) a 3-D FE analysis, and (2) a 1-D homogenized beam analysis. The results of both analysis showed that the overall flexural Young's modulus of the unit cell was significantly smaller than the extensional modulus. In addition, no noticeable coupling between the bending-extension responses and the shear-extension responses was observed. Although less rigorous and accurate than the full 3-D FE simulation, the simple 1-D homogenized beam analysis provided reasonable estimates for the longitudinal extensional and flexural moduli.

Using Eq. (31) (Whitcomb et al, 1998) and the FEA results, the effective flexural stiffness for a multi-ply laminate was calculated as a function of the number of plies, $n$. The ratio of the flexural to extensional Young's moduli is plotted in Figure 5.1 for the longitudinal and transverse directions. The plot shows that for $n=4$ plies, the extensional moduli approximates the flexural moduli within $5 \%$. For $n<4$ plies, the two homogenized moduli differ considerably.

$$
\bar{E}_{f}=\frac{E_{f}}{n^{2}}+E\left(1-\frac{1}{n^{2}}\right)
$$

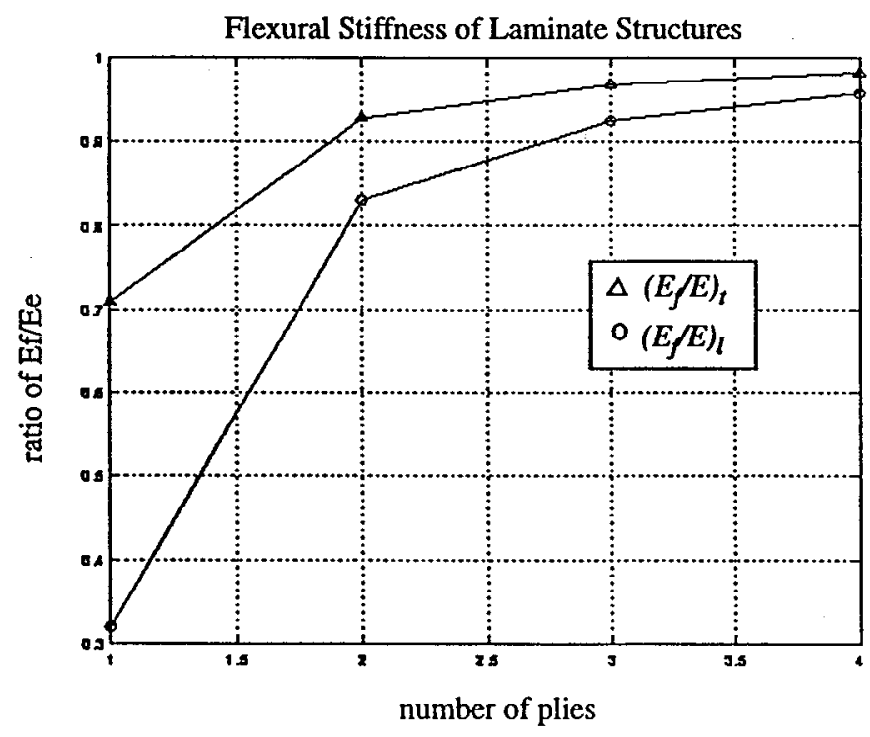

Figure 5.1 The flexural stiffness of a laminate structure as a function of the number of plies

In general, the elastic response of a braided laminate structure with more than four plies can be accurately represented using only the effective extensional properties. However, to model a braided laminate made with only a few plies or a section of a delaminated braided structure, distant lamina-level extensional and flexural properties are necessary to accurately characterize its elastic response. The primary physical reason for this is that in braided systems the fibers undulate through the entire lamina thickness, and, consequently, 
the fibers are non-uniformly distributed, in the through-thickness direction, through out the RVE.

The behavior and modeling requirements for braided composites differ from those for traditional unidirectional composites. Because the fibers are relatively straight in unidirectional systems, many essentially identical, relatively small, RVEs are distributed uniformly through the thickness of a lamina. As noted by Pagano (1974) and Whitcomb et al (1998), when many RVEs exist through the thickness of a structure, the structure's effective flexural moduli approximately equals the RVE's effective extensional moduli. Consequently, only the effective extensional properties are needed to accurately represent the elastic behavior a laminate, a single lamina, or even a small portion of a lamina in unidirectional systems.

\section{Acknowledgments}

The authors wish to acknowledge the support of Dr. Joseph Carpenter and the U.S. Department of Energy's Partnership for the Next Generation Vehicle program. 


\section{Reference}

Dadkhah, M. S., Flintoff, J. G., Kniveton, T., and Cox, B. N., 1995, "Simple Models for Triaxially Braided Composites", Composites, v. 26, pg. 564.

Maker, B.N, 1995, NIKE3D: A Nonlinear, Implicit, Three-Dimensional Finite Element Code for Solid and Structural Mechanics-User's Manual, Lawrence Livermore National Laboratory UCRL-MA-105268 Rev. 1.

Marrey, V. R. and Sankar, V. B., 1997, "A Micromechanical Model for Textile Composite Plates", Journal of Composite Materials, v. 31, pg. 1201.

Naik, A. R., Ifju, P. G, and Masters, E. J., 1994, "Effects of Fiber Architecture Parameters on Deformation Fields and Elastic Moduli of 2-D Braided Composites", Journal of Composite Materials, v. 29, pg. 658.

Pagano, N. J., 1974, Mechanics of Composite Materials, eds., G. P. Sendeckyg, Academic Press, New York, v. 2, pg. 1-44.

Vandeurzen, P., Ivens, J., and Verpoest, I., 1997, “TEXCOMP: A 3D Analysis Tool for 2D Woven Fabric Composites", SAMPE Journal, v. 33, pg. 25-33.

Whitcomb, J.D, Chapman, C. D., and Srirengan, K., 1998, “Analysis of Plain Weave Composites Subjected to Flexure", Texas A\&M University - Aerospace Engineering Report, submitted for publication. 